\title{
Accuracy of Preoperative Local Staging of Primary Colorectal Cancer by Using Computed Tomography: Reappraisal Based on Data Collected at a Highly Organized Cancer Center
}

\author{
Jung Sub So, Chinock Cheong, Seung Yeop Oh, Jei Hee Lee ${ }^{1}$, Young Bae Kim², Kwang Wook Suh \\ Departments of Surgery, ${ }^{1}$ Radiology, and ${ }^{2}$ Pathology, Ajou University School of Medicine, Suwon, Korea
}

Purpose: In patients with colorectal cancer, preoperative staging using various imaging technologies is important for establishing the treatment plan and predicting the prognosis. Although computed tomography (CT) has been used most widely, the versatility of CT accuracy was primarily because of the lack of specialization. In this study, we aimed to identify whether any advancement in abdominal CT accuracy in the prediction of local staging has occurred.

Methods: Between December 2014 and November 2015, patients with colorectal cancer were retrospectively enrolled. All CT findings were retrospectively reported. A total of 285 patients were included, and their retrospectively collected data were retrospectively reviewed, focusing on a comparison between preoperative and postoperative staging.

Results: The overall prediction accuracy of the T stage was $55.1 \%$, with overstaging occurring in $63(22.1 \%)$ and understaging in 65 patients (22.8\%). The sensitivity and specificity were $90.0 \%$ and $68.4 \%$, respectively. The overall prediction accuracy of the $\mathrm{N}$ stage was $54.7 \%$, with overstaging occurring in 89 (31.2\%) and understaging in 40 patients (14.1\%). The sensitivity and specificity were $71.9 \%$ and $63.2 \%$, respectively. The CT accuracies by pathologic stage were $0 \%, 62.2 \%$, $25.3 \%$, and $81.2 \%$ for stages 0 (Tis N0), I, II, and III, respectively.

Conclusion: CT has good sensitivity for detecting colon cancers with tumor invasion beyond the bowel wall. However, detection of nodal involvement using CT is unreliable. In our opinion, abdominal CT alone has limitations in predicting the local staging of colorectal cancer, and additional technologies, such as CT plus positron emission tomography and/or colonography, will improve its accuracy.

Keywords: Colorectal neoplasms; TNM staging; Computed tomography

\section{INTRODUCTION}

Colorectal cancer (CRC) is the third most common malignancy in Western countries [1]. In Korea, its incidence is increasing in the aging population, even though CRC-related mortality remains

Received: July 19, 2017 • Accepted: September 15, 2017

Correspondence to: Kwang Wook Suh, M.D.

Department of Surgery, Ajou University School of Medicine, 206 World cup-ro, Yeongtong-gu, Suwon 16499, Korea

Tel: +82-31-219-7833, Fax: +82-31-219-5755, E-mail: suhkw@aumc.ac.kr

(c) 2017 The Korean Society of Coloproctology

This is an open-access article distributed under the terms of the Creative Commons Attribution NonCommercial License (http://creativecommons.org/licenses/by-nc/4.0) which permits unrestricted noncommercial use, distribution, and reproduction in any medium, provided the original work is properly cited. unchanged [2]. The treatment for patients with CRC involves surgical removal of the primary tumor, with an adequate margined segment of the bowel together with regional lymph nodes. Contiguous invasion toward adjacent organs is a frequent finding in patients with CRC. Even in this situation, combined removal of the involved organs has been proven to be curative. In addition, adjuvant chemotherapy is the standard treatment modality for patients with stage III and stage II disease.

In patients with CRC, preoperative staging using various imaging technologies is important for establishing the treatment plan and predicting the patient's prognosis [3, 4]. Among others, computed tomography (CT) has been the most widely used tool owing to its advantages of being universally available and easily reproducible [5]. The most prominent feature in preoperative stag- 
ing using CT in patients with CRC is the detection of distant metastases [6]. Numerous studies have attempted to identify the accuracy of preoperative CT in terms of predicting the extents of tumor invasion and regional lymph node metastases. However, the results of those studies are varied.

In our opinion, the versatility of CT accuracy is primarily because of the lack of specialization [7]. Therefore, we hypothesize that radiologists, surgeons, and pathologists who are highly organized in CRC management can use CT to arrive at a relatively more accurate preoperative staging. We have been collaborating as a CRC team since 2012. In our hospital, a radiologist checks the colonoscopy findings before reading the CT images and participates in the weekly multidisciplinary conference, joining physicians, pathologists, and surgeons. In the present study, we aimed to identify if any advancement in the accuracy of abdominal CT in the prediction of local staging has taken place.

\section{METHODS}

The present study was conducted to assess the ability of CT staging to predict accurately the depth of tumor invasion and lymph node involvement in patients diagnosed with CRC. Consecutive patients who underwent elective curative surgery for nonmetastatic CRC at our department of surgery between December 2014 and November 2015 were retrospectively enrolled. In our colorectal cancer center, the multidisciplinary team consists of colorectal surgeons, physicians, pathologists and radiologist, and about 500 surgeries are performed on patients for CRC, not including benign neoplasms, each year. Two surgeons whose sole clinical expertise is colorectal surgery and who have been in practice for longer than 15 years perform all surgical procedures at our colorectal cancer center. All the patients had a biopsy-proven adenocarcinoma. The patients with recurrent disease or metastatic disease were excluded from the study cohort. All CT findings were retrospectively reported. Ultimately, 285 patients were included in the present analysis. This study was reviewed and approved by the appropriate Institutional Review Board, along with a waiver of informed consent (AJIRB-MED-SUR-01-0017).

\section{Preoperative staging}

The preoperative clinical evaluation included physical examination, colonoscopy, abdominopelvic CT (APCT), chest CT, complete blood-cell-count test, liver function test, and serum carcinoembryonic antigen level assessment. We used CT scans and the 7th Union for International Cancer Control (UICC) TNM staging system to assess the radiological stage. The $\mathrm{T}$ stages were defined as follows: T1, tumor invades submucosa; T2, tumor invades muscularis propria; $\mathrm{T} 3$, tumor invades through the muscularis propria into pericolorectal tissues; $\mathrm{T} 4 \mathrm{a}$, tumor penetrates to the surface of the visceral peritoneum; and T4b, tumor directly invades or is adherent to other organs or structures $[8,9]$. The $\mathrm{N}$ stages were defined as follows: N0, no regional lymph node me- tastases; N1, metastasis in 1-3 regional lymph nodes; and N2, metastasis in four or more regional lymph nodes [9].

Multidetector computed tomography (MDCT) was performed using one of the following CT scanners: Sensation 16 (Siemens Healthcare, Forchheim, Germany) $(\mathrm{n}=10)$, SOMATOM Definition Flash (Siemens Healthcare, Forchheim, Germany) $(n=22)$, Brilliance 16 (Philips Healthcare, Cleveland, OH, USA) $(n=11)$, or Brilliance 64 (Philips Healthcare) $(n=20)$. From the portal venous phase scan, the transverse image data sets were reconstructed with a 3-mm section thickness. Further, we also reformatted the coronal images multiplanar reformation images with a 3-mm section thickness.

The scanning procedures were performed while the patient held his or her breath. After scanning the CT scout view in the supine position, contrast-enhanced images were obtained with the patients in the same position. Contrast-enhanced CT was conducted after intravenous injection of an iodinated contrast agent, iopromide (Ultravist 300, Schering, Berlin, Germany); $120 \mathrm{~mL}$ of the contrast agent was administered at a rate of $3 \mathrm{~mL} / \mathrm{sec}$. CT was conducted during the portal venous phase (start delay of $60 \mathrm{sec}-$ onds) and delayed phase (start delay of 180 seconds). The scanning parameters were as follows: $120 \mathrm{kVp}, 120-160 \mathrm{mAs}, 512 \times$ 512 matrix, and $2.5-\mathrm{mm}$ reconstruction thickness in $1-\mathrm{mm}$ reconstruction intervals. The average acquisition time was $15 \mathrm{sec}-$ onds. The evaluation began with the 2-dimensional transverse CT images, followed by 3-dimensional CT colonography. We considered a lymph node to be positive if the longest diameter was $>1.0 \mathrm{~cm}$ or if it was 0.7 to $1.0 \mathrm{~cm}$ in size and showed strong enhancement, round shape, central necrosis, or perinodal infiltration, all of which suggest a metastasis. A specialist in colorectal imaging who was blinded to the surgical pathological findings interpreted or reviewed all the CT images.

\section{Surgery and pathologic staging}

All the patients underwent a standard colectomy or a total mesorectal excision and regional lymphadenectomy according to the tumor's location. A curative resection was defined as the event when no gross residual tumor remained in the surgical bed and the proximal and the distal resection margins were pathologically negative for tumor invasion. The pathological staging was also determined by using the final pathological features according to the 7th UICC TNM staging system and positron emission tomography (PET) scanning, if available [9].

\section{Statistical analysis}

Statistical evaluation was performed using IBM SPSS Statistics ver. 20.0 (IBM Co., Armonk, NY, USA). Continuous variables were analyzed using the analysis of variance test, and data are presented as means (ranges). Categorical variables were analyzed using the chi-square test or Fisher exact test. We also calculated the sensitivity and the specificity for preoperative $\mathrm{CT}$ imaging. A P $\leq$ 0.05 was deemed to be statistically significant. 


\section{Coloproctology Jung Sub so, et al.}

Table 1. Patients' characteristics $(\mathrm{n}=285)$

\begin{tabular}{|c|c|}
\hline Variable & Value \\
\hline Age (yr) & $65.3(41-90)$ \\
\hline \multicolumn{2}{|l|}{ Sex } \\
\hline Male & $159(55.8)$ \\
\hline Female & $126(44.2)$ \\
\hline Body mass index $\left(\mathrm{kg} / \mathrm{m}^{2}\right)$ & $23.7(17.1-32.4)$ \\
\hline History of abdominal surgeries & $73(25.6)$ \\
\hline Preoperative chemoradiotherapy & $13(4.6)$ \\
\hline \multicolumn{2}{|l|}{ Tumor site } \\
\hline Ascending colon & $83(29.1)$ \\
\hline Transverse colon & $11(3.9)$ \\
\hline Descending colon & $8(2.8)$ \\
\hline Sigmoid colon & $114(40.0)$ \\
\hline Rectum & $65(22.8)$ \\
\hline Synchronous tumor & $4(1.4)$ \\
\hline \multicolumn{2}{|l|}{ Tumor location of rectal cancer } \\
\hline Upper (>15 cm) & $16(24.6)$ \\
\hline Mid (5.1-15 cm) & $24(36.9)$ \\
\hline Low $(\leq 5 \mathrm{~cm})$ & $25(38.5)$ \\
\hline \multicolumn{2}{|l|}{ Operations } \\
\hline Right hemicolectomy & 89 (31.2) \\
\hline Transverse colectomy & $3(1.1)$ \\
\hline Left hemicolectomy & $12(4.2)$ \\
\hline Anterior resection & $49(17.2)$ \\
\hline Low anterior resection & 95 (33.3) \\
\hline Hartmann's operation & $13(4.6)$ \\
\hline Abdominoperineal resection & $14(4.9)$ \\
\hline Subtotal or total colectomy & $10(3.6)$ \\
\hline \multicolumn{2}{|l|}{ Surgical method } \\
\hline Open & $130(45.6)$ \\
\hline Laparoscopic \& robot & $155(54.4)$ \\
\hline Preoperative CEA (ng/mL) & $21.1(0.6-3,487)$ \\
\hline Tumor size (cm) & $4.5(0.2-17.5)$ \\
\hline \multicolumn{2}{|l|}{ Histologic grade } \\
\hline Well-differentiated & $41(14.4)$ \\
\hline Moderately differentiated & $198(69.5)$ \\
\hline Poorly differentiated & $9(3.2)$ \\
\hline Others (mucinous, signet ring cell, etc.) & $37(13.0)$ \\
\hline No. of harvested LNs & $21(3-93)$ \\
\hline No. of positive LNs & $1.48(0-22)$ \\
\hline
\end{tabular}

Values are presented as median (range) or number (\%). CEA, carcinoembryonic antigen; LN, lymph node.

\section{RESULTS}

This study included 285 consecutive patients with CRC who had undergone curative surgery. Two colorectal surgeons whose sole clinical expertise is colorectal surgery and who have been in practice for longer than 15 years performed all surgical procedures. The study cohort included 159 men (55.8\%), and the median age of the 285 patients was 65.3 years (range, $41-90$ years). Of the 285 patients, $83(29.1 \%)$ presented with ascending colon cancer, 11 (3.9\%) with transverse colon cancer, 8 (2.8\%) with descending colon cancer, 114 (40.0\%) with sigmoid colon cancer, 65 (22.8\%) with rectal cancer, and 4 (1.4\%) with synchronous cancer; 130 (45.6\%) underwent conventional open surgery and 155 (54.4\%) underwent laparoscopy-assisted surgery. The median number of resected lymph nodes was 21 (range, 3-93). The other demographic and pathologic features are summarized in Table 1.

The overall accuracy in the prediction of the $\mathrm{T}$ stage was $55.1 \%$, with overstaging occurring in $63(22.1 \%)$ and understaging in 65 patients $(22.8 \%)$. The sensitivity was $90.0 \%$, and the specificity was $68.4 \%$ (Table 2). The accuracy by depth of invasion was $37.1 \%$ for $\mathrm{T} 1,24 \%$ for $\mathrm{T} 2,67.3 \%$ for $\mathrm{T} 3$, and $43.3 \%$ for $\mathrm{T} 4$ lesions. The accuracy in predicting T3 and T4 lesions was significantly higher than that in predicting $\mathrm{T} 1$ and $\mathrm{T} 2$ lesions $(\mathrm{P}<0.001)$. The overall accuracy in the prediction of the $\mathrm{N}$ stage was $54.7 \%$, with overstaging occurring in $89(31.2 \%)$ and understaging in 40 patients (14.1\%). The sensitivity was $71.9 \%$, and the specificity was $63.2 \%$ (Table 3 ). The accuracy by subgroup of the $\mathrm{N}$ stage was

Table 2. Correlation between CT and pathologic preoperative staging in patients with colorectal cancer according to the $\mathrm{T}$ stage

\begin{tabular}{lrrrrrr}
\hline \multirow{2}{*}{ CT T stage } & \multicolumn{7}{c}{ Pathologic T stage, $\mathrm{n}$} \\
\cline { 2 - 7 } & T0 & T1 & T2 & T3 & T4 & Total \\
\hline T0 & 14 & 10 & 11 & 16 & 4 & 55 \\
T1 & 1 & 13 & 2 & 0 & 0 & 16 \\
T2 & 7 & 8 & 8 & 9 & 0 & 32 \\
T3 & 3 & 4 & 11 & 109 & 13 & 140 \\
T4 & 0 & 0 & 1 & 28 & 13 & 42 \\
Total & 25 & 35 & 33 & 162 & 30 & 285 \\
\hline
\end{tabular}

$\mathrm{CT}$, computed tomography.

Table 3. Correlation between CT and pathologic preoperative staging in patients with colorectal cancer according to the $\mathrm{N}$ stage

\begin{tabular}{lrrrr}
\hline \multirow{2}{*}{ CT N stage } & \multicolumn{4}{c}{ Pathologic N stage, $\mathrm{n}$} \\
\cline { 2 - 5 } & N0 & N1 & N2 & Total \\
\hline N0 & 108 & 24 & 8 & 140 \\
N1 & 42 & 30 & 8 & 80 \\
N2 & 21 & 26 & 18 & 65 \\
Total & 171 & 80 & 34 & 285 \\
\hline
\end{tabular}

CT, computed tomography. 
Table 4. Correlation between CT staging and pathologic preoperative staging in patients with colorectal cancer according to the TNM stage

\begin{tabular}{lcrcccc}
\hline \multirow{2}{*}{ CT TNM stage } & \multicolumn{6}{c}{ Pathologic TNM stage, $\mathrm{n}$} \\
\cline { 2 - 7 } & Stage 0 & Stage I & Stage II & Stage III & Stage IV & Total \\
\hline Stage 0 & 14 & 19 & 7 & 13 & 1 & 54 \\
Stage I & 7 & 23 & 6 & 5 & 1 & 42 \\
Stage II & 1 & 3 & 25 & 14 & 1 & 44 \\
Stage III & 3 & 10 & 45 & 72 & 3 & 133 \\
Stage IV & 0 & 0 & 3 & 5 & 4 & 12 \\
Total & 25 & 55 & 86 & 109 & 10 & 285 \\
\hline
\end{tabular}

CT, computed tomography.

$63.2 \%$ for N0, 37.5\% for N1, and 52.9\% for N2. The accuracy in predicting $\mathrm{N} 0$ and $\mathrm{N} 2$ lesions was significantly higher than that in predicting $\mathrm{N} 1$ lesions $(\mathrm{P}<0.001)$. The $\mathrm{CT}$ accuracy by pathologic stage was $0 \%$ for stage 0 (Tis N0), $62.2 \%$ for stage I, $25.3 \%$ for stage II, and $81.2 \%$ for stage III (Table 4 ).

\section{DISCUSSION}

Preoperatively, a complete evaluation of patients with CRC includes staging of the primary tumor and evaluation of distant metastases. Because CT is the most useful diagnostic modality in terms of feasibility, some studies advocate and support the use of abdominal CT or APCT for local staging of CRC for treatment planning and selection of patients for neoadjuvant treatment [5]. Although magnetic resonance imaging (MRI) and endorectal ultrasound (EUS) have been proven to be more accurate than CT, they are currently not routinely used for staging CRC $[5,10]$. Some studies examined the feasibility of using MRI to stage colon cancers; however, the technique is limited to the detection of lesions rather than local staging [11]. EUS is also used to predict the depth of tumor invasion in patients with rectal cancer, but requires organized techniques, instruments, and expertise, which are not routinely available, to access colon cancers $[5,12,13]$.

This study aimed to determine the diagnostic accuracy of abdominal CT for staging CRC and to evaluate whether CT can help in differentiating high-risk patients from low-risk patients. The overall accuracies of abdominal CT in predicting the $\mathrm{T}$ and the $\mathrm{N}$ stages of resectable CRC were $55.1 \%$ and $54.7 \%$, respectively. The results showed that CT is more accurate when tumor invasion beyond the bowel wall exists, with an accuracy of $67.3 \%$ for T3 and $43.3 \%$ for T4 lesions. An explanation could be that to minimize the risk of understaging, radiologists interpret minimal pericolonic fat stranding owing to benign desmoplastic reaction as tumor invasion. This is a well-known problem in CRC staging. A relatively low specificity of abdominal CT is a commonly found result. When reviewing the literature, study designs were found to influence the results; retrospective studies showed a higher sensi- tivity, but had a much lower specificity, than prospective studies.

The predictive power of $\mathrm{N}$ staging using abdominal CT was also unsatisfactory. In our study, the overall accuracy was less than $70 \%$, and the specificity was only $63.2 \%$. The sensitivity of $\mathrm{N}$ stage prediction was higher in studies using a slice thickness of $<5 \mathrm{~mm}$ than in studies using a slice thickness of $\geq 5 \mathrm{~mm}$ (78\% vs. $62 \%$, respectively). Thin slices $(<5 \mathrm{~mm})$ improve the assessment of various criteria, such as the size, shape, and border contour of the lymph node, probably by reducing the partial volume effect. However, the specificity remained almost the same regardless of the slice thickness. Therefore, the observation that lymph node diameter per se is not a reliable indicator of nodal metastasis in patients with CRC is acceptable. Even in the analysis using MDCT techniques, the specificity was still low (69\%). This could be explained by the fact that a greater number of small lymph nodes are detected using thin slices, which is beneficial; however, small lymph nodes are more difficult to characterize and might be overstaged more frequently by radiologists while trying to avoid understaging, thus increasing the sensitivity, but decreasing the specificity.

Attempts have been made to improve the staging in colon cancers by combining functional imaging techniques, such as PET, with MDCT. Studies have shown that PET/CT colonography can be used as a single technique for the diagnosis and staging of colon cancer with an improved accuracy $[11,14]$. Another novel functional MRI technique uses diffusion-weighted imaging (DWI) based on the ability to depict movements of water molecules in tissues [15]. The magnitude of the water molecule's movement is expressed as an apparent diffusion coefficient (ADC) value. The $\mathrm{ADC}$ value of metastatic lymph nodes in patients with $\mathrm{CRC}$ is found to be significantly lower than that in patients with nonmetastatic lymph nodes. Previous studies comparing the accuracy between DWI MRI and PET/CT in the staging of colon cancer showed that although PET/CT was better at local staging of colon cancer, the detection of malignant lymph nodes was better with DWI MRI $[16,17]$.

The results of this prospective analysis show that even in a highly organized center, abdominal CT is still inaccurate in the prediction of preoperative staging of CRC. Our results show that the main limitation of CT is its inability to identify metastatic lymph nodes accurately and to differentiate true tumors from peritumoral inflammations or adenomas.

In conclusion, CT has a good sensitivity for the detection of colon cancers with tumor invasion beyond the bowel wall. However, detecting nodal involvement using CT is unreliable. These findings were similar for both colon cancer and rectal cancer. In our opinion, abdominal CT alone has unsolved limitations in the prediction of local staging of CRC. As pieces of evidence suggest, additional technologies, such as CT plus PET and/or colonography, will improve the accuracy of CT. 


\section{CONFLICT OF INTEREST}

No potential conflict of interest relevant to this article was reported.

\section{REFERENCES}

1. GLOBOCAN 2012. Estimated cancer incidence, mortality and prevalence worldwide in 2012 [Internet]. Lyon: International Agency for Research on Cancer; 2015 [cited 2015 Dec 9]. Available from: http://globocan.iarc.fr/Default.aspx.

2. Oh CM, Won YJ, Jung KW, Kong HJ, Cho H, Lee JK, et al. Cancer statistics in Korea: incidence, mortality, survival, and prevalence in 2013. Cancer Res Treat 2016;48:436-50.

3. Thoeni RF, Rogalla P. Current CT/MRI examination of the lower intestinal tract. Baillieres Clin Gastroenterol 1994;8:765-96.

4. Thoeni RF. Colorectal cancer. Radiologic staging. Radiol Clin North Am 1997;35:457-85.

5. Elmas N, Killi RM, Sever A. Colorectal carcinoma: radiological diagnosis and staging. Eur J Radiol 2002;42:206-23.

6. Hundt W, Braunschweig R, Reiser M. Evaluation of spiral CT in staging of colon and rectum carcinoma. Eur Radiol 1999;9:78-84.

7. Thoeni RF, Rogalla P. CT for the evaluation of carcinomas in the colon and rectum. Semin Ultrasound CT MR 1995;16:112-26.

8. Sobin LH, Gospodarowicz MK, Wittekind C. TNM classification of malignant tumours. Hoboken (NJ): Wiley-Blackwell; 2009.

9. Edge SB, Byrd DR, Compton CC, Fritz AG, Greene FL, Trotti A, editors. AJCC cancer staging manual. 7th ed. New York: Springer; 2010.
10. Maldjian C, Smith R, Kilger A, Schnall M, Ginsberg G, Kochman M. Endorectal surface coil MR imaging as a staging technique for rectal carcinoma: a comparison study to rectal endosonography. Abdom Imaging 2000;25:75-80.

11. Kijima S, Sasaki T, Nagata K, Utano K, Lefor AT, Sugimoto H. Preoperative evaluation of colorectal cancer using CT colonography, MRI, and PET/CT. World J Gastroenterol 2014;20:16964-75.

12. Glaser F, Schlag P, Herfarth C. Endorectal ultrasonography for the assessment of invasion of rectal tumours and lymph node involvement. Br J Surg 1990;77:883-7.

13. Pappalardo G, Reggio D, Frattaroli FM, Oddi A, Mascagni D, Urciuoli $\mathrm{P}$, et al. The value of endoluminal ultrasonography and computed tomography in the staging of rectal cancer: a preliminary study. J Surg Oncol 1990;43:219-22.

14. de Haan MC, van Gelder RE, Graser A, Bipat S, Stoker J. Diagnostic value of CT-colonography as compared to colonoscopy in an asymptomatic screening population: a meta-analysis. Eur Radiol 2011;21:1747-63.

15. Pham TT, Liney GP, Wong K, Barton MB. Functional MRI for quantitative treatment response prediction in locally advanced rectal cancer. Br J Radiol 2017;90:20151078.

16. Will O, Purkayastha S, Chan C, Athanasiou T, Darzi AW, Gedroyc W, et al. Diagnostic precision of nanoparticle-enhanced MRI for lymph-node metastases: a meta-analysis. Lancet Oncol 2006;7:5260.

17. Figueiras RG, Goh V, Padhani AR, Naveira AB, Caamaño AG, Martin CV. The role of functional imaging in colorectal cancer. AJR Am J Roentgenol 2010;195:54-66. 